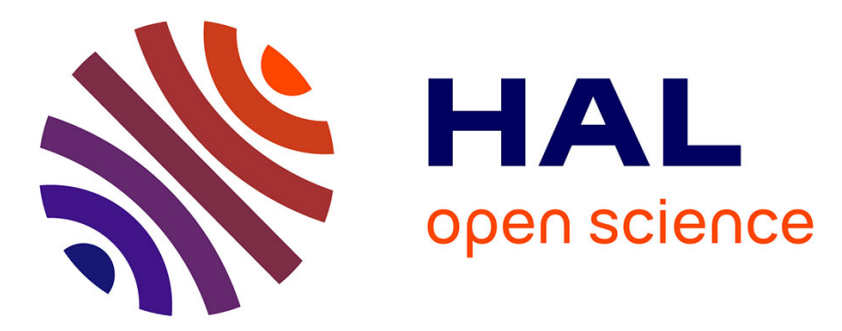

\title{
Relations génétiques entre les résistances par non-acceptation et par antibiose du melon à Aphis gossypii. Recherche de liaisons avec d'autres gènes
}

\author{
Michel Pitrat, Hervé Lecoq
}

\section{To cite this version:}

Michel Pitrat, Hervé Lecoq. Relations génétiques entre les résistances par non-acceptation et par antibiose du melon à Aphis gossypii. Recherche de liaisons avec d'autres gènes. Agronomie, 1982, 2 (6), pp.503-508. 10.1051/agro:19820601 . hal-02728366

\section{HAL Id: hal-02728366 \\ https: / hal.inrae.fr/hal-02728366}

Submitted on 2 Jun 2020

HAL is a multi-disciplinary open access archive for the deposit and dissemination of scientific research documents, whether they are published or not. The documents may come from teaching and research institutions in France or abroad, or from public or private research centers.
L'archive ouverte pluridisciplinaire HAL, est destinée au dépôt et à la diffusion de documents scientifiques de niveau recherche, publiés ou non, émanant des établissements d'enseignement et de recherche français ou étrangers, des laboratoires publics ou privés. 


\title{
Relations génétiques entre les résistances par non-accep- tation et par antibiose du melon à Aphis gossypii. Recherche de liaisons avec d'autres gènes
}

\author{
Michel PITRAT \& Hervé LECOQ* \\ avec la collaboration technique de Monique RICARD \\ I.N.R.A., Station d'Amélioration des Plantes maraîchères, \\ (*) Station de Pathologie végétale, Centre de Recherches d'Avignon BP 94, F 84140 Montfavet.
}

\section{RÉSUMÉ}

Cucumis melo,

Cucurbitacées,

Puceron,

Aphididae,

Hérédité,

Liaison génétique,

Homoptera,

Transmission de virus.

\begin{abstract}
La résistance à la colonisation des plantes de melon (Cucumis melo L.) par Aphis gossypii Glov. peut être assurée par plusieurs mécanismes. Nous étudions les relations entre la non-acceptation (= non-préférence) et l'antibiose en testant chaque plante dans des générations en disjonction $F_{2}$ et $R_{1}$ pour les 2 caractères : nombre de pucerons restant $24 \mathrm{~h}$ après le dépôt de 10 adultes (non-acceptation) et nombre de larves pondues en 3 j par 4 adultes dans des cages fixées aux feuilles (antibiose). Il apparaît que les plantes résistantes par non-acceptation sont également résistantes par antibiose. Le même allèle contrôlant également la résistance à la transmission des virus par A. gossypii, nous proposons de le nommer Virus aphid transmission resistance et de le symboliser par Vat. Ce gène est indépendant de Fom-1, Fom-2, Wmv-1 et ms-1 mais il est lié au gène Fn
\end{abstract} $(11,6 \pm 1,9$ unités de recombinaison).

\author{
SUMMARY \\ Cucumis melo, \\ Cucurbitaceae \\ Aphid, \\ Aphididae, \\ Inheritance, \\ Linkage, \\ Homoptera, \\ Virus transmission.
}

\begin{abstract}
Genetic relations between non-acceptance and antibiosis resistance to Aphis gossypii in melon. Search for linkage with other genes

Resistance to colonization of melon (Cucumis melo L.) by Aphis gossypii Glov. can arise through several mechanisms. We have studied the relations between non-acceptance (= non-preference) and antibiosis by assaying all the plants of $\mathrm{F}_{2}$ and $\mathrm{BC}_{1}$ progenies between PI 414723 (resistant) and Doublon (susceptible) for both mechanisms : this was done by counting the number of aphids remaining $24 \mathrm{~h}$ after 10 adults were placed on a plant (non-acceptance) and the number of larvae produced in 3 days by 4 adults in small leaf cages (antibiosis). Plants which were resistant by non-acceptance also exhibited antibiosis. Since the same gene also governs resistance to virus transmission by $A$. gossypii, we propose for it the name Virus aphid transmission resistance and the symbol Vat. This gene is independent of Fom-1, Fom-2, Wmv-1 and ms-1 but is linked to Fn $(11.6 \pm 1.9)$ units.
\end{abstract}

\section{INTRODUCTION}

Le puceron du melon (Aphis gossypii Glov.) peut avoir un double effet néfaste sur les cultures de melon (Cucumis melo L.). C'est un déprédateur qui, à la suite du développement de colonies, peut provoquer des dégâts graves (enroulement des feuilles, arrêt de la croissance) pouvant aller jusqu'à la mort de la plante. En général, les prédateurs et les parasites ainsi que quelques traitements aphicides permettent de contrôler les populations du puceron. Cependant, il est surtout dangereux comme vecteur de virus. $A$. gossypii peut transmettre au melon sur le mode non persistant les virus de la mosaïque du concombre (CMV), de la mosaïque de la pastèque 1 et 2 (WMV 1 et WMV 2) et du rabougrissement jaune du melon (MYSV). C'est le puceron le plus fréquemment rencontré dans les cultures de melon du Sud-Est de la France et l'un des meilleurs vecteurs des virus cités.

$\mathrm{Au}$ cours d'un programme de recherche de résistance du melon au CMV, nous avons mis en évidence un nouveau mécanisme de résistance : la résistance à la transmission des virus par $A$. gossypii (LECOQ et al., 1979). Cette résistance est spécifique du puceron $A$. gossypii mais elle est active visà-vis de tous les virus étudiés (LECOQ et al., 1980 ; RISSER et al., 1981). Le contrôle génétique de la résistance à la transmission est assuré par un allèle dominant (PITRAT \& LECOQ, 1980). La présence de cet allèle entraîne également la résistance à la colonisation des plantes par $A$. gossypii suivant un mécanisme de «non-préférence" (PAINTER, 1951). Les termes de «non-acceptance » (VAN MARREWIJK $\&$ de PONTI 1975) et «antixenosis» (KOGAN \& ORTMAN, 1978) ont également été proposés pour définir ce mode de 
résistance. Nous utiliserons "non-acceptation", traduction française de «non-acceptance », qui nous paraît le mieux décrire le phénomène : l'insecte n'accepte pas la plante pour s'alimenter et se reproduire, même en l'absence de choix.

KISHABA et al. (1971) ont étudié la résistance du melon à A. gossypii. Ils ont montré que les 3 mécanismes de résistance décrits par PAINTER existaient chez la lignée $\mathrm{LJ}$ 90234 (BOHN et al., 1972) et ils ont étudié l'hérédité de la tolérance et de l'antibiose. La tolérance caractérisée par le non enroulement des feuilles est contrôlée par un allèle dominant symbolisé par Ag (BoHN et al., 1973). Un gène majeur partiellement dominant confère l'antibiose (mesurée par le nombre de larves produites pendant un certain temps) mais des gènes mineurs peuvent modifier le niveau d'antibiose (KISHABA et al., 1976).

Nous apprécions le niveau de non-acceptation, qui est lié à la résistance à la transmission des virus, par le nombre de pucerons restant sur la plante $24 \mathrm{~h}$ après le dépôt de 10 adultes (PITRAT \& LeCOQ, 1980). Mais ce n'est pas un phénomène de "tout ou rien " et nous avons voulu savoir si les quelques pucerons restant sur une plante étaient capables de coloniser cette plante en pondant de nombreuses larves. Une $1^{\text {re }}$ série d'expériences a donc pour but d'étudier les relations entre 2 mécanismes de résistance à $A$. gossypii : la non-acceptation et l'antibiose.

Dans la $2^{\mathrm{c}}$ série d'expériences, nous avons voulu savoir s'il existait des liaisons entre le gène gouvernant la nonacceptation et la résistance à la transmission des virus et d'autres gènes contrôlant les résistances à des maladies ou la stérilité mâle. En effet, dans un programme de sélection, nous sommes amenés à cumuler les résistances à plusieurs maladies et il nous a paru utile de savoir s'il y avait indépendance ou liaison entre ces gènes.

\section{MATÉRIEL ET MÉTHODES}

La technique utilisée pour l'élevage d'A. gossypii sur melon a été décrite par LECOQ et al. (1979). Les graines de melon sont semées dans du sable puis les plantules sont repiquées en pots dans un mélange de terreau et de tourbe. Le test de non-acceptation (PITRAT \& LECOQ, 1980) a lieu lorsque la $1^{\text {re }}$ feuille commence à s'étaler, soit 15 à $20 \mathrm{j}$ après le semis. Après ce test, les plantes sont traitées par fimigation avec un aphicide (sulfotep) et gardées en serre pendant 1,5 à 2 mois avant d'être soumises à un essai d'antibiose. L'antibiose est mesurée par le nombre de larves produites en $3 \mathrm{j}$ par 4 jeunes adultes aptères dans une petite cage fixée à la face inférieure d'une feuille. Sur chaque plante, 2 feuilles adultes sont testées, chacune avec une cage. Cette étude a été réalisée sur des descendants du croisement entre "Doublon" (lignée sensible de type «Cantaloup Charentais ») et «PI 414723 » (lignée résistante d'origine indienne).

Dans la $2^{c}$ série d'expériences, après le traitement aphicide suivant le test de non-acceptation, les plantes ont été conservées pour observer la fertilité pollinique ou bien ont été contaminées avec l'un des agents pathogènes suivants:

- souche FOM 26 appartenant au pathotype 1 de Fusarium oxysporum Schl. f. sp. melonis Sn. et Hans. (RISSER et al., 1976),

- souche DAVIS 5 appartenant au pathotype 2 de F. oxysporum f. sp. melonis,

- souche E9 du pathotype F du MYSV (LECOQ et al., 1981),

- souche E2 du WMV 1.

Les inoculations de $F$. oxysporum f. sp. melonis sont réalisées par immersion des pots dans une suspension de conidies du champignon. Les inoculations de virus (WMV 1 et MYSV) sont faites par inoculation mécanique suivant la méthode couramment utilisée dans notre laboratoire (MARROU, 1967).

Différentes lignées de melon ont été utilisées suivant les liaisons étudiées :

- Liaison Vat-Fom-1 : «Doublon» $(+$ Fom-1) et «PI 414723 " $($ Vat +$)$,

- Liaison Vat-Fom-2 : «Doublon» $(++)$ et «PI 161375 " (Vat Fom-2),

— Liaison Vat-Wmv-1 : «72025» $(+W m v-1)$ et «PI $161375 »($ Vat +$)$,

- Liaison Vat-ms-1 : lignée de sélection $(+m s-1)$ et «PI $161375 »($ Vat +$)$,

- Liaison Vat-Fn : «Doublon» $(+F n)$ et «PI $161375 »$ $(V a t+)$.

\section{RÉSULTATS}

\section{A. Relation entre la non-acceptation et l'antibiose}

Nous avons étudié les descendances de croisements entre «PI 414723 » et «Doublon» (tabl. 1). La lignée de «PI 414723 ", étudiée par BoHN \& KishaBA sous la dénomination « LJ 90234 », est résistante à $A$. gossypii par nonacceptation et antibiose (BOHN et al., 1972).

Les valeurs des tests de non-acceptation et d'antibiose observées sur la $F_{1}$ sont supérieures à celles du parent résistant "PI 414723 » et surtout les variances sont plus élevées. Dans les générations en disjonction $F_{2}$ et $R_{1}$ $\left(F_{1} \times\right.$ «Doublon»), la plupart des plantes se comportent

\section{TABLEAU 1}

Résultats des tests de non-acceptation et d'antibiose vis-à-vis d'A. gossypii sur 2 lignées de melon et leur hybride $F_{l}$. Non-acceptance and antibiosis assays to A. gossypii on 2 muskmelon lines and their $F_{1}$ hybrid.

\begin{tabular}{|c|c|c|c|c|c|c|}
\hline & \multicolumn{3}{|c|}{ Non-acceptation } & \multicolumn{3}{|c|}{ Antibiose } \\
\hline & Moyenne & $\begin{array}{c}\text { Erreur } \\
\text { type }\end{array}$ & $\begin{array}{l}\text { Valeurs } \\
\text { extrêmes }\end{array}$ & Moyenne & $\begin{array}{l}\text { Erreur } \\
\text { type }\end{array}$ & $\begin{array}{l}\text { Valeurs } \\
\text { extrêmes }\end{array}$ \\
\hline «Doublon » & 9,7 & 0,13 & $7-10$ & 100,5 & 2,53 & $75,5-120,5$ \\
\hline «PI $414723 »$ & 3,3 & 0,28 & $1-7$ & 4,9 & 0,85 & $0-19,5$ \\
\hline$F_{1}(«$ Doublon $» \times \ll P I 414723 »)$ & 6,2 & 0,39 & $1-10$ & 17,5 & 2,25 & $1-56$ \\
\hline
\end{tabular}


TABLEAU 2

Résultats des tests de non-acceptation et d'antibiose sur 9 plantes $F_{2}$ supposées être des recombinants et sur les lignées $F_{3}$ issues de ces plantes $F_{2}$. Non-acceptance and antibiosis assays on $9 F_{2}$ plants supposed to be recombinants and on the $F_{3}$ progenies from these $F_{2}$ plants.

\begin{tabular}{|c|c|c|c|c|c|c|c|c|c|}
\hline & & \multicolumn{2}{|c|}{ Test sur plante $F_{2}$} & \multicolumn{6}{|c|}{ Test sur descendance $\mathrm{F}_{3}$} \\
\hline & & \multirow{2}{*}{$\begin{array}{c}\text { Non- } \\
\text { acceptation }\end{array}$} & \multirow{2}{*}{ Antibiose } & \multicolumn{3}{|c|}{ Non-acceptation } & \multicolumn{3}{|c|}{ Antibiose } \\
\hline & & & & Moyenne & $\begin{array}{c}\text { Erreur } \\
\text { type }\end{array}$ & $\begin{array}{l}\text { Valeurs } \\
\text { extrêmes }\end{array}$ & Moyenne & $\begin{array}{l}\text { Erreur } \\
\text { type }\end{array}$ & $\begin{array}{l}\text { Valeurs } \\
\text { extrêmes }\end{array}$ \\
\hline \multirow[t]{9}{*}{ («Doublon $» \times \ll$ P $1414723 »)$} & $1 \mathrm{~A}$ & 5 & 85 & 9,6 & 0,12 & $7-10$ & 90,4 & 2,84 & $50-108$ \\
\hline & $1 \mathrm{D}$ & 1 & 128 & 9,6 & 0,12 & $7-10$ & 79,6 & 1,97 & $60-103$ \\
\hline & $1 \mathrm{~K}$ & 4 & 74,5 & 4,2 & 0,42 & $1-10$ & 8,2 & 1,41 & $2-31$ \\
\hline & $1 \mathrm{H}$ & 5 & 58 & 5,6 & 0,52 & $2-10$ & 9,5 & 3,33 & $0-73,5$ \\
\hline & $1 \mathrm{~B}$ & 9 & 1,5 & 3,4 & 0,37 & $0-7$ & 13,1 & 1,78 & $0-39$ \\
\hline & $1 \mathrm{~F}$ & 9 & 11 & 2,9 & 0,26 & $1-7$ & 8,6 & 1,09 & $0-37$ \\
\hline & $1 G$ & 10 & 5,5 & 4,7 & 0,34 & $0-10$ & 5,2 & 0,69 & 0-18 \\
\hline & $1 \mathrm{C}$ & 10 & 10 & 4,9 & 0,66 & $0-10$ & 30,3 & 6,76 & $1,5-107$ \\
\hline & $1 E$ & 8 & 5,5 & 4,3 & 0,52 & $0-10$ & 15,9 & 3,90 & $0-91$ \\
\hline
\end{tabular}

comme les parents c'est-à-dire sont sensibles par nonacceptation et antibiose ou résistantes par ces 2 mécanismes (fig. 1). Cependant, parmi les 149 plantes $F_{2}$ étudiées, quelques-unes pourraient être considérées comme des «recombinants». 4 plantes $F_{2}$ résistantes par non-acceptation et sensibles par antibiose (1A, $1 \mathrm{D}, 1 \mathrm{H}$ et $1 \mathrm{~K}$ du tabl. 2) et 5 plantes sensibles par acceptation et résistantes par antibiose $(1 \mathrm{~B}, 1 \mathrm{C}, 1 \mathrm{E}, 1 \mathrm{~F}$ et $1 \mathrm{G})$ ont été conservées et autofécondées. Les descendances $F_{3}$ de ces 9 plantes $F_{2}$ ont été testées suivant la même technique.

Les résultats résumés dans le tableau 2 indiquent que :

- 3 descendances $\mathrm{F}_{3}(1 \mathrm{C}, 1 \mathrm{E}$ et $1 \mathrm{H})$ sont en disjonction pour la non-acceptation et l'antibiose. Elles correspondaient à des plantes $F_{2}$ hétérozygotes et nous avons déjà signalé que les plantes $F_{1}$ ont un niveau de résistance moins élevé et surtout un comportement plus variable que le parent résistant (fig. 1).

- 5 descendances $F_{3}(1 \mathrm{~A}, 1 \mathrm{~B}, 1 \mathrm{D}, 1 \mathrm{~F}$ et $1 \mathrm{G})$ sont homogènes pour la non-acceptation et l'antibiose et présentent le comportement de la plante $F_{2}$ pour le test d'antibiose, c'est-à-dire sont résistantes (1B, 1F et 1G) ou sensibles (1A et 1D).

- 1 descendance $F_{3}(1 \mathrm{~K})$ est homogène et résistante pour la non-acceptation et l'antibiose, c'est-à-dire a le comportement du test de non-acceptation de la plante $F_{2}$.

Les 9 plantes $F_{2}$ conservées ne peuvent donc pas être considérées comme des recombinants. Les 6 descendances $\mathrm{F}_{3}$ homogènes pour la non-acceptation et l'antibiose résulteraient, lors de l'infestation de la $\mathrm{F}_{2}$, d'imperfections ou d'échappées au test de non-acceptation ( 5 cas sur 149) ou au test d'antibiose (1 cas sur 149).

Nous pouvons conclure que la présence de l'allèle gouvernant la non-acceptation entraîne une résistance par antibiosc. Cet allèle contrôle également la résistance à la transmission des virus par $A$. gossypii (PITRAT \& LECOQ, 1980). Nous proposons de le nommer Virus aphid transmission resistance et de le symboliser par Vat.

\section{TABLEAU 3}

Répartition des phénotypes observés sur des générations en disjonction pour l'allèle Vat et Fom-1, Fom-2, Wmv-1, ms-1 ou Fn. Observed segregations of $F_{2}$ and $B C_{1}$ progenies for Vat and Fom-1, Fom-2, Wmv-1, ms-1 or Fn.

\begin{tabular}{|c|c|c|c|c|c|c|c|c|}
\hline & \multirow{2}{*}{$\begin{array}{l}\text { Nombre } \\
\text { plantes } \\
\text { observées }\end{array}$} & \multicolumn{4}{|c|}{ Phénotype } & \multirow{2}{*}{$\begin{array}{l}\text { Disjonction } \\
\text { testée }\end{array}$} & \multirow{2}{*}{$\begin{array}{c}\chi^{2} \\
\text { calculé }\end{array}$} & \multirow{2}{*}{$\begin{array}{l}\text { Probabilité } \\
\text { du } \chi^{2}\end{array}$} \\
\hline & & $\begin{array}{l}\text { Double } \\
\text { dominant }\end{array}$ & $\begin{array}{c}\text { Dominant } \\
\text { récessif }\end{array}$ & $\begin{array}{c}\text { Récessif } \\
\text { dominant }\end{array}$ & $\begin{array}{l}\text { Double } \\
\text { récessif }\end{array}$ & & & \\
\hline $\mathrm{F}_{2}\left(\frac{V^{\prime} a t+}{+ \text { Fom-1 }}\right) \oplus$ & 234 & 135 & 50 & 38 & 11 & $9: 3: 3: 1$ & 2,627 & $0,30-0,50$ \\
\hline $\mathrm{F}_{2}\left(\frac{\text { Vat Fom }-2}{++}\right) \oplus$ & 288 & 158 & 52 & 58 & 20 & $9: 3: 3: 1$ & 0,691 & $0,75-0,50$ \\
\hline $\mathrm{F}_{2}\left(\frac{V a l+}{\overline{W m v-1}}\right) \oplus$ & 297 & 166 & 45 & 63 & 23 & $9: 3: 3: 1$ & 4,079 & $0,20-0,30$ \\
\hline$V_{2}\left(\frac{V a t+}{+m s-I}\right) \oplus$ & 276 & 144 & 46 & 66 & 20 & $9: 3: 3: 1$ & 5,816 & $0,10-0,20$ \\
\hline $\mathrm{F}_{2}\left(\frac{V a t+}{\overline{+F n}}\right) \oplus$ & 276 & 204 & 3 & 11 & 58 & $9: 3: 3: 1$ & 189,585 & $<1 \%$ \\
\hline $\mathrm{RC}_{1}\left(\frac{V a t+}{\overline{+F_{n}}}\right) \times(++)$ & 293 & 19 & 123 & 136 & 15 & $1: 1: 1: 1$ & 174,044 & $<1 \%$ \\
\hline
\end{tabular}


DOUBLON

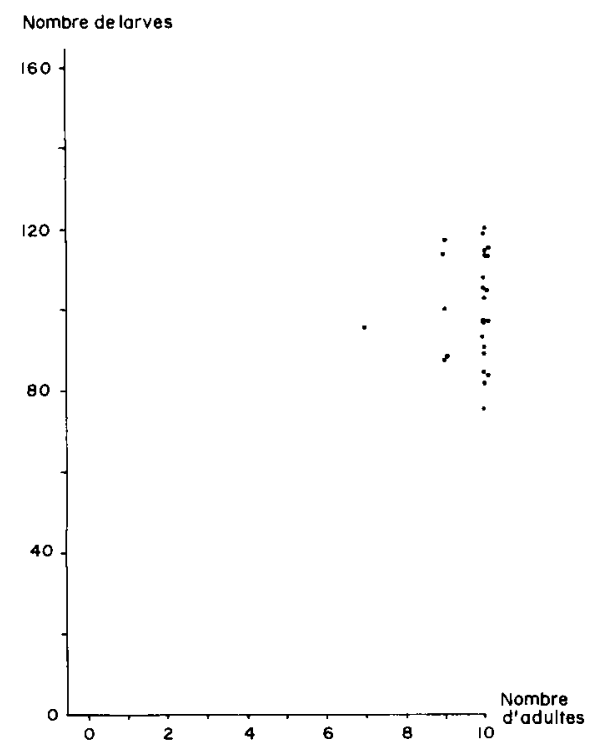

$F_{1}:$ DOUBLON $\times$ PI 414723
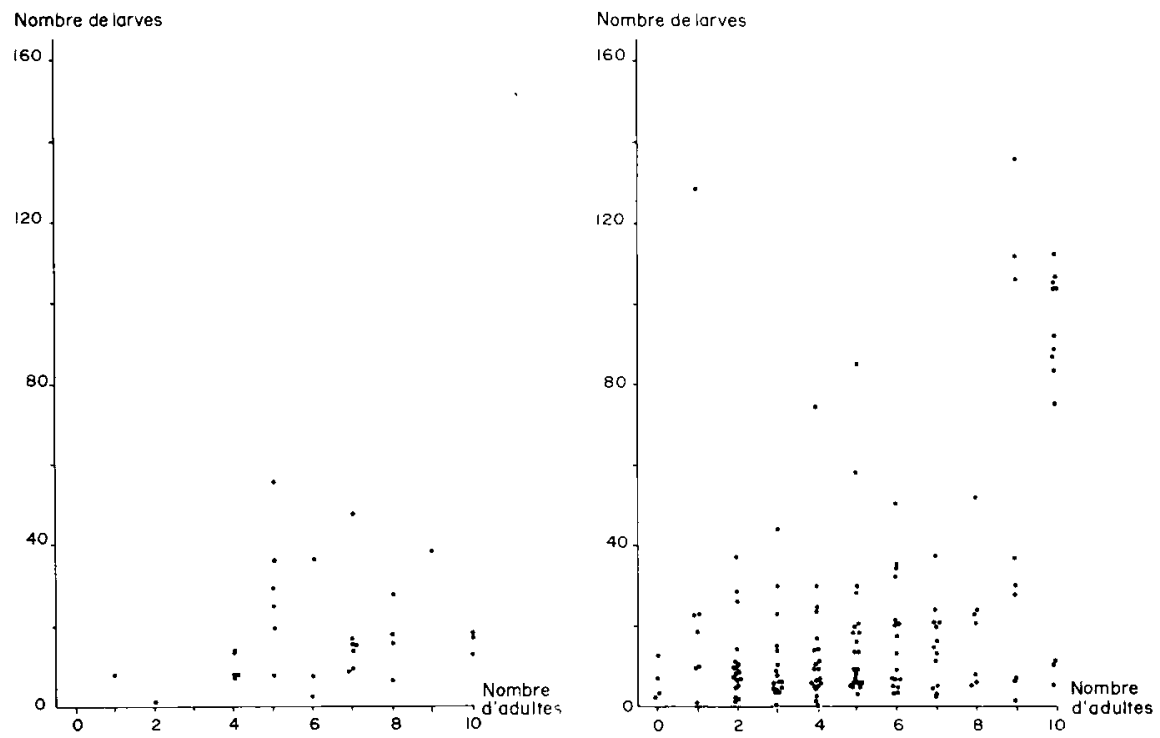

Figure 1

Relation entre la non-acceptation (nombre d'adultes restant sur les plantes) et l'antibiose (nombre de larves produites dans une cage) vis$\grave{a}$-vis d'Aphis gossypii chez le melon.

Relation between non-acceptance (number of adults remaining on the plants) and antibiosis (number of larvae produced in a leaf-cage) to Aphis gossypii in melon.

Les résultats rapportés dans le tabieau 3 montrent que Vat est indépendant de Fom-1, Fom-2, Wmv-1 et ms-1. Par contre, il existe une liaison entre Vat et $F n$. Le pourcentage de recombinaison, estimé sur la $F_{2}$ suivant la méthode du maximum de vraisemblance, est égal à $p=5,3$ p. 100 (erreur type 6,0) et, calculé sur le recroisement de la $F_{1}$ par le double récessif, le pourcentage de recombinaison est de $\mathrm{p}=11,6 \mathrm{p} .100$ (erreur type 1,9).

\section{DISCUSSION ET CONCLUSIONS} entraine le flétrissement et la mort des plantes après inoculation de pathotype $\mathrm{F}$ du MYSV alors que les plantes $\mathrm{Fn}^{+} / \mathrm{Fn}^{+}$présentent des symptômes de jaunissement, éclaircissement des nervures et arrêt de la croissance (RISSER et al., 1981).
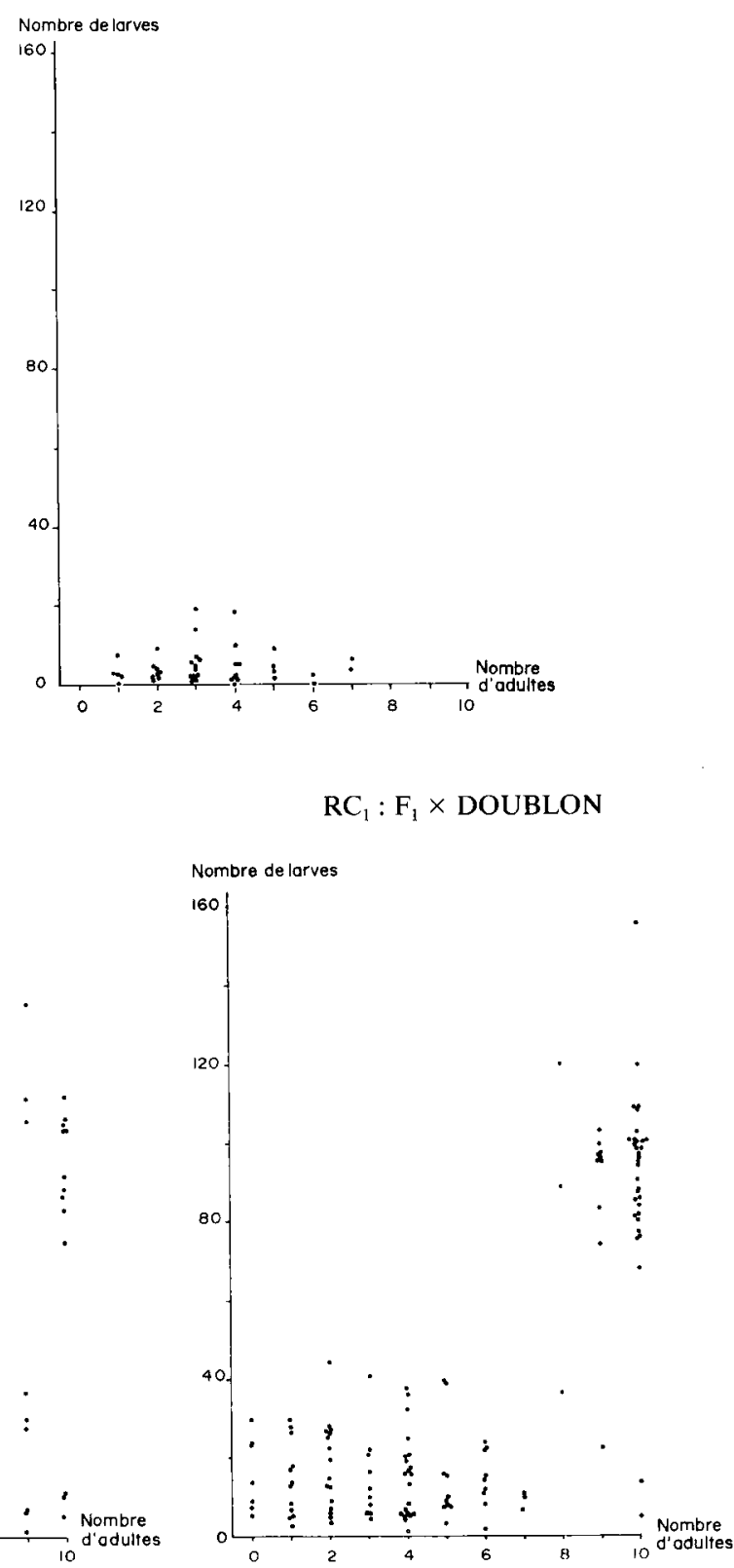

$\mathrm{RC}_{1}: \mathrm{F}_{1} \times$ DOUBLON au WMV 1 (WEBB, 1979); la présence de l'allèle $F n$ disjonction pour l'allèle Vat et l'allèle $m s-1$ de stérilité mâle (BOHN \& WHITAKER, 1949) ou un allèle de résistance à un autre agent pathogène. L'allèle Fom-1 apporte la résistance aux pathotypes 0 et 2 de $F$. oxysporum f. sp. melonis et l'allèle Fom-2 la résistance aux pathotypes 0 et 1 (RISSER et l'allèle 1976); l'allèle $W m v-1$ la résistance par hypersensibilité 
contrôlées montre que 2 mécanismes de résistance interviennent. La non-acceptation d'une plante de melon par A. gossypii s'exprime par le refus du puceron à rester sur la płante. Si l'on oblige les pucerons à rester sur une feuille d'une plante résistante au moyen de petites cages, le nombre de larves pondues est considérablement réduit par rapport à une plante sensible (antibiose).

D'après KISHABA et al. (1976), l'antibiose est contrôlée par un gène majeur partiellement dominant et des gènes mineurs modificateurs. Nous montrons ici que ce gène majeur est identique au gène gouvernant la non-acceptation et la résistance à la transmission des virus par $A$. gossypii (PITRAT \& LecoQ, 1980). Nous proposons de le nommer Virus aphid transmission resistance et de le symboliser par $V a t$. Nous ne savons pas quelles sont les relations entre Vat et le gène $A g$ qui contrôle la tolérance (absence d'enroulement des feuilles) (BoHN et al., 1973). Pratiquement, en un seul test qui dure $24 \mathrm{~h}$, le sélectionneur peut choisir les plantes résistantes à $A$. gossypii par non-acceptation et antibiose et résistantes à la transmission des virus.

Environ 45 gènes ont été décrits chez le melon mais peu de liaisons ont été mises en évidence (RoBinson et al., 1976 ; RoBINSON, 1979). ZINK (1977) a observé un pourcentage de recombinaison de 19,4 entre « yellow virescent " et «bush ». Les gènes Fom-1, Fom-2, Wmv-1 et $m s-1$ sont indépendants de $V a t$ et nous savons que la résistance oligogénique au CMV n'est pas liée à Vat (PITRAT \& LECOQ, 1980). Il ne devrait donc pas y avoir de difficultés pour cumuler dans une même variété les résistances à $A$. gossypii, aux races 0,1 et 2 de $F$. oxysporum $\mathrm{f}$. $\mathrm{sp}$. melonis, au CMV et au WMV 1 ainsi que la stérilité mâle. Par contre, il existe une liaison entre Vat et $F n$ qui a pu être estimée à 11,6 unités de recombinaison. Les 7 variétés de melon résistantes à $A$. gossypii ( PI 161375 ", «PI 164320», «PI 255478 », «PI 414723 », « Ginsen makuwa », «Kanro makuwa» et «Shiroubi okayama») ne flétrissent pas après inoculation mécanique avec le pathotype $\mathrm{F}$ de MYSV et possèdent vraisemblablement l'allèle $\mathrm{Fn}^{+}$(RISSER et al., 1981).

Reçu le 14 septembre 1981. Accepté le 12 février 1982.

\section{RÉFÉRENCES BIBLIOGRAPHIQUES}

Bohn G. W., Kishaba A. N., Principe J. A., Toba H. H., 1973. Tolerance to melon aphid in Cucumis melo. J. am. Soc. hortic. Sci., 98, 37-40.

Bohn G. W., Kishaba A. N., Toba H. H., 1972. Mechanisms of resistance to melon aphid in a muskmelon line. Hortscience, 7, 281282.

Bohn G. W., Whitaker T. W., 1949. A gene for male sterility in the muskmelon (Cucumis melo). Proc. am. Soc. hortic. Sci., 53, 308314.

Kishaba A. N., Bohn G. W., Toba H. H., 1971. Resistance to Aphis gossypii in muskmelon. J. econ. Entomol., 64, 935-937.

Kishaba A. N., Bohn G. W., Toba H. H., 1976. Genetic aspects of antibiosis to Aphis gossypii in Cucumis melo from India. J. am. Soc. hortic. Sci., 101, 557-561.

Kogan M., Ortman E. F., 1978. Antixenosis a new term proposed to define Painter's « non-preference " modality of resistance. Bull. entomol. Soc. Am., 24, 175-176.

Lecoq H., Cohen S., Pitrat M., Labonne G., 1979. Resistance to cucumber mosaic virus transmission by aphids in Cucumis melo. Phytopathology, 69, 1223-1225.

Lecoq H., Labonne G., Pitrat M., 1980. Specificity of resistance to virus transmission by aphids in Cucumis melo. Ann. Phytopathol., 12, $139-144$.

Lecoq H., Pitrat M., Clement M., 1981. Identification et caractérisation d'un potyvirus provoquant la maladie du rabougrissement jaune du melon. Agronomie, 1, 10, 827-834.
Marrou J., 1967. Amélioration des méthodes de transmission mécanique des virus par absorption des inhibiteurs d'infection sur le charbon végétal. C.R. Acad. Agric. Fr., 53, 972-981.

Painter R. H., 1951. Insect resistance in crop plants. Macmillan Co. New York, $520 \mathrm{pp}$.

Pitrat M., Lecoq H., 1980. Inheritance of resistance to cucumber mosaic virus transmission by Aphis gossypii in Cucumis melo. Phytopathology, 70, 958-961.

Risser G., Banihashemi Z., Davis D. W., 1976. A proposed nomenclature of Fusarium oxysporum f. sp. melonis races and resistance genes in Cucumis melo. Phytopathology, 66, 1105-1106.

Risser G., Pitrat M., Lecoq H., Rode J. C., 1981. Sensibilité variétale du melon au virus du rabougrissement jaune du melon et à sa transmission par Aphis gossypii. Hérédité de la réaction de flétrissement. Agronomie, 1, 10, 835-838.

Robinson R. W., 1979. New genes for the Cucurbitaceac. Rep. Cucurbit Genet. Coop., 2, 49-53.

Robinson R. W., Munger H. M., Whitaker T. W., Bohn G. W., 1976. Genes of the Cucurbitaceae. Hortscience, 11, 554-568.

Van Marrewijk G. A. M., de Ponti O. M. B., 1975. Possibilities and limitations of breeding for pest resistance. Meded. Fak. Landbouw. Rijks. Univ. Gent, 40, 229-247.

Webb R. E., 1979. Inheritance of resistance to watermelon mosaic virus 1 in Cucumis melo L. Hortscience, 14, 265-266.

Zink F., 1977. Linkage of virescent foliage and plant growth habit in muskmelon. J. am. Soc. hortic. Sci., 102, 613-615. 